\title{
TIDAL CORRECTIONS FOR PRECISE GRAVITY MEASUREMENTS IN IZU PENINSULA
}

\author{
Hideo HANADA* \\ Earthquake Research Institute, University of Tokyo, Tokyo, Japan \\ (Received June 18, 1979)
}

\begin{abstract}
Effects of earth and oceanic tides on the Earth's gravitational constant, hereinafter called gravity for short, are estimated for precise gravity measurements in Izu peninsula. In calculating the oceanic effects, we apply Green's function given by Farrell to hourly tidal records obtained at the nearby tidal stations and to the tidal charts given by Tiron et al. As a result, the oceanic effect ranges from about 5 to $10 \mu \mathrm{gal}$ in the peninsula. This fact may lead to the conclusion that hereafter, the oceanic tidal correction seems to be necessary for precise gravity measurements. The optimal tidal factor of gravity ( $\mathrm{G}$-factor) and phase lag used in the earth tidal corrections are estimated as 1.15 with a phase lag of 0.1 hour, although we have usually adopted a G-factor of 1.20 without any phase lag. The difference between gravity values corrected with the usual tidal constants and ones estimated in the present study amounts to about $5 \mu$ gal.
\end{abstract}

\section{Introduction}

It is said that the accuracy of gravity measurements should be within $10 / \mathrm{gal}$ for monitoring the vertical crustal movements (TsuboKaWA, 1970), therefore, the corrections should be made to gravity values within an accuracy of $10 \mu \mathrm{gal}$. There are many disturbing factors on gravity, such as earth tides, oceanic tides, atmospheric pressure, and ground water level, whose effects seem to be beyond several $\mu \mathrm{gal}$. The effects of atmospheric pressure and ground water on gravity have been studied (NAKAI, 1975; LAMBert and Beaumont, 1977; HaGIWARA, 1977), although there still remain ambiguous parts from a theoretical aspect.

The effects of the oceanic tides on gravity have also been treated so far in several reports (NAKagaWA, 1962; SATO, 1975; TANAKA, 1977, 1978; Tsukamoto and NAKAGAWA, 1979). Judging from their results, the oceanic tidal effects may possibly amount to $10 \mu \mathrm{gal}$. OsHIma and YoKoYAMA (1977) have recently reported the oceanic effects in the coastal

* Present address: International Latitude Observatory of Mizusawa, Mizusawa, Japan. 
region of Sakurajima and showed that it exceeds $20 \mu$ gal. The above facts imply that, if we estimate exactly the effects of oceanic tides on gravity, the detection of gravity change resulting from land-deformation can be made more precisely.

It is also necessary for a precise gravity survey to estimate the value of the tidal factor of gravity (G-factor) at various stations, because a difference of 10 per cent in the G-factor results in an error of about 10 $\mu$ gal. In the conventional way of earth tidal corrections made in Japan, the value of the $\mathrm{G}$-factor has been supposed to be 1.20 and that of phase lag to be 0 hour, although these values are not presumed to be optimal. In this paper, the effects of oceanic tides on gravity will be estimated for correcting gravity data obtained in Izu peninsula. In addition, the optimal values of the G-factor and phase lag in Izu peninsula will be proposed.

\section{Methods of Oceanic Tidal Corrections}

Oceanic effects on gravity consist of two principal parts. The first is the direct attraction of sea water ('attraction part') and the second is an effect from the elastic deformation of the solid earth ('elastic part') due to the load to sea water (FARRELL, 1972). In order to calculate the oceanic effects on gravity, the total region of the sea is divided into a region within about $120 \mathrm{~km}$ distance of the observation stations in Izu peninsula ('near region') and another region outside it ('far region'). Both

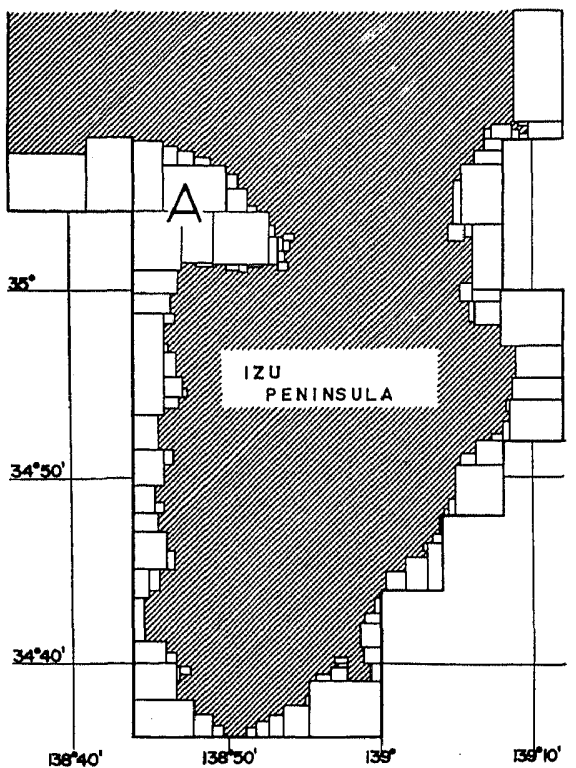

Fig. 1. Divisions of ' $A$ ' region. 

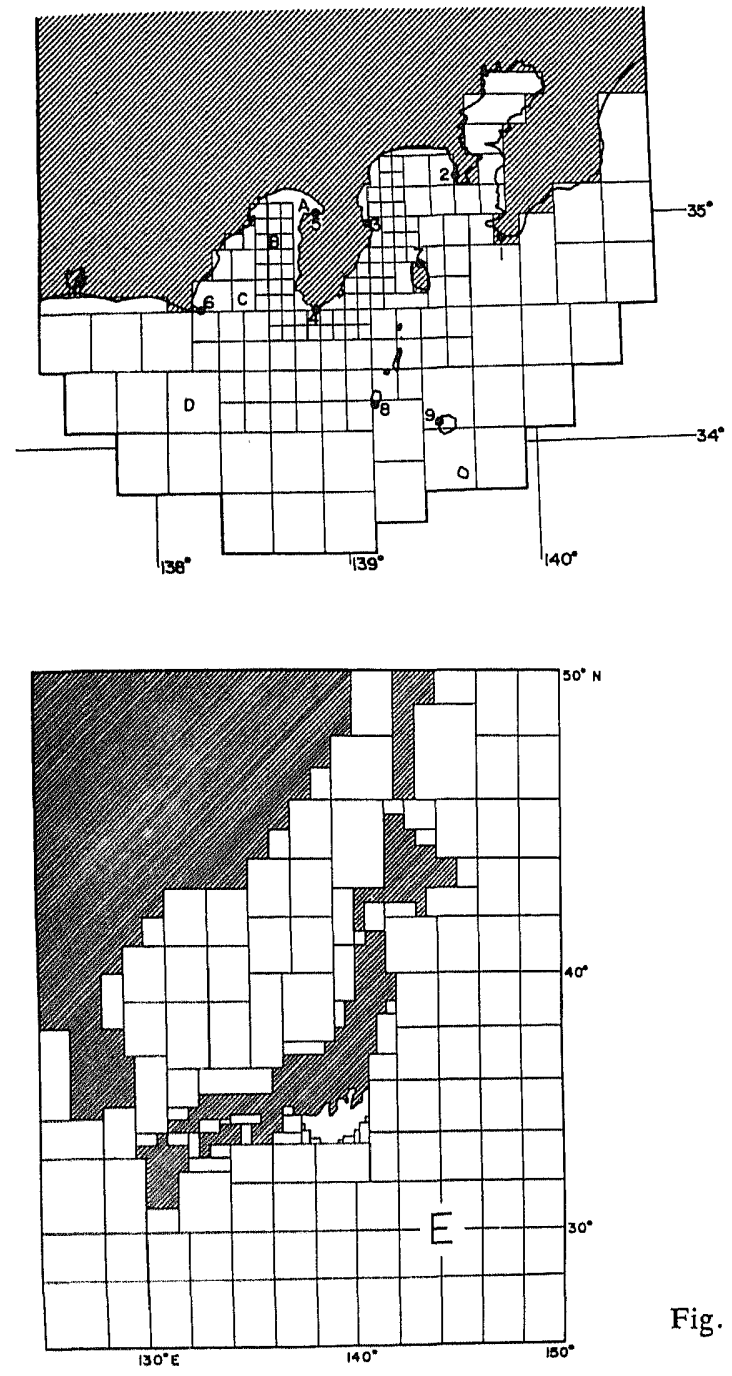

Fig. 2. Divisions of ' $B$ ', ' $C$ ' and ' $D$ ' regions. Solid circles indicate the locations of tidal stations. 1, Mera; 2, Aburatsubo; 3, Ito; 4, Minami-izu; 5, Uchiura; 6, Omaezaki; 7, Okada; 8, Kozushima; 9, Miyakejima.

Fig. 3. Divisions of ' $E$ ' region.

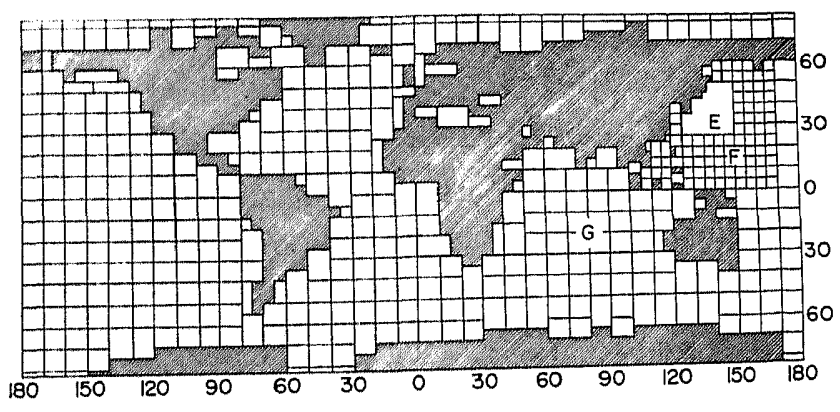

Fig. 4. Divisions of ' $F$ ' and ' $G$ ' regions. 
regions are further divided into smaller-sized rectangular meshes, and they are classified into seven groups by their dimensions.

As a whole, an area of the mesh is approximately proportional to the square of its distance from the observation stations in order that the oceanic effect from each mesh may be equal, since the attraction part per unit area is in inverse proportion to the square of the distance. ' $A$ ' region is the nearest region of the observation station and the meshes contained there are the smallest (Fig. 1). Particularly, the meshes adjacent to the coastline are made as small as possible in order that the oceanic effect at a station near the coast may be estimated precisely. According to the increase in distance from the station, the regions are named ' $B$ ', ' $C$ ', ' $D$ ', ' $E$ ', ' $F$ ' and ' $G$ ' and the meshes contained in each region have dimension of $4^{\prime} \times 4^{\prime}, 8^{\prime} \times 8^{\prime}, 16^{\prime} \times 16^{\prime}, 2^{\circ} \times 2^{\circ}, 5^{\circ} \times 5^{\circ}$ and $10^{\circ} \times 10^{\circ}$, respectively, as shown in Figs. 2, 3 and 4 . In such a way, 'A', ' $B$ ', ' $C$ ' and ' $D$ ' cover the near region and ' $E$ ', ' $F$ ' and ' $G$ ' the far region.

The near-region sea surface can be approximated to a plain ("plain surface model') so that a surface integral can be calculated analytically. The attraction part $\left(g^{\mathrm{A}}\right)$ at an observation point $\mathrm{O}$ generated by a water prism as shown in Fig. 5 can be written as follows:

$$
\begin{aligned}
g^{\mathrm{A}}= & G \rho \int_{\zeta_{1}}^{\zeta_{2}} \int_{\eta_{1}}^{\eta_{2}} \int_{\xi_{1}}^{\xi_{2}} \frac{\zeta}{\left(\sqrt{\left.\xi^{2}+\eta^{2}+\zeta^{2}\right)^{3}} \mathrm{~d} \xi \mathrm{d} \eta \mathrm{d} \zeta\right.} \\
= & -G \rho\left\{\xi \log \left(\sqrt{\xi^{2}+\eta^{2}+\zeta^{2}}+\eta\right)+\eta \log \left(\sqrt{\left.\xi^{2}+\eta^{2}+\zeta^{2}+\xi\right)}\right.\right. \\
& \left.-\xi+2|\zeta| \tan ^{-1} \frac{\sqrt{\xi^{2}+\eta^{2}+\zeta^{2}}+\xi+\eta}{|\zeta|}\right\}|| \mid,
\end{aligned}
$$

where $\rho$ is density of sea water, $G$ the Newtonian gravitation constant and

$$
\begin{aligned}
& \xi_{i}=x_{i}-x_{0}, \\
& \eta_{i}=y_{i}-y_{0}, \\
& \zeta_{i}=z_{i}-z_{0},
\end{aligned}
$$

for $i=1$ and 2 . The notation $\|$ in (1) is defined as follows:

$$
\begin{aligned}
f(\xi, \eta, \zeta) \mid \|= & f\left(\xi_{2}, \eta_{2}, \zeta_{2}\right)-f\left(\xi_{1}, \eta_{2}, \zeta_{2}\right)-f\left(\xi_{2}, \eta_{1}, \zeta_{2}\right)+f\left(\xi_{1}, \eta_{1}, \zeta_{2}\right) \\
& -f\left(\xi_{2}, \eta_{2}, \zeta_{1}\right)+f\left(\xi_{1}, \eta_{2}, \zeta_{1}\right)+f\left(\xi_{2}, \eta_{1}, \zeta_{1}\right)-f\left(\xi_{1}, \eta_{1}, \zeta_{1}\right) .
\end{aligned}
$$

In case of a plain surface model, the attraction part generated by a water layer mass can be obtained by summing up (1) over all the meshes in the near region. Now, we compare the attraction part of oceanic effects calculated by making use of a plain surface model with that of a spherical surface model (the near-region sea surface is approximated to a 


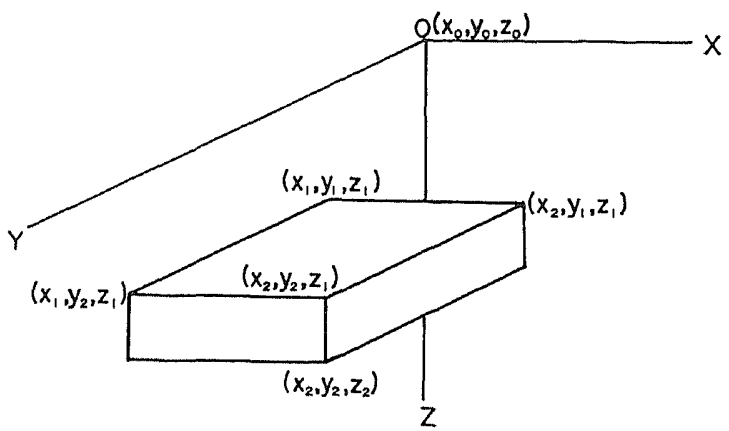

Fig. 5. A rectangular prismatic water body and the coordinate system used in the calculations of oceanic effect on gravity.

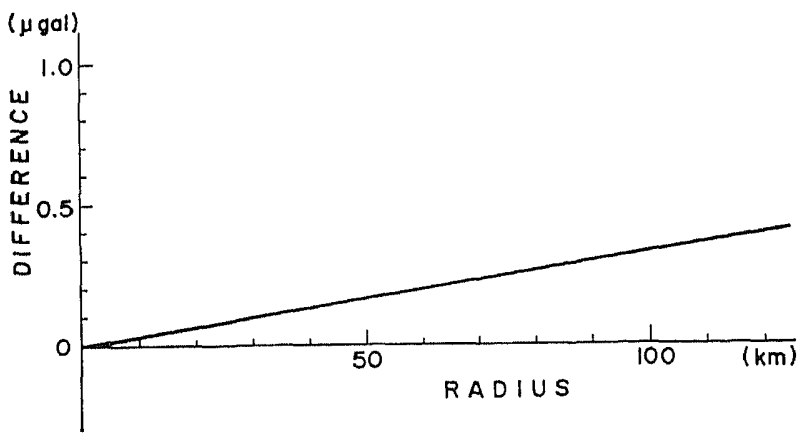

Fig. 6. Differences between the oceanic effects (attraction part) calculated by making use of a plain surface model and that of a spherical surface model. We assume here the attraction is generated by a circular water layer of $1 \mathrm{~m}$ thickness and of $120 \mathrm{~km}$ radius and the observation point is supposed to be $100 \mathrm{~m}$ above the center of the circular water layer.

spherical surface). Figure 6 indicates the differences between them on the assumption that the attraction is generated by a circular water layer of $1 \mathrm{~m}$ thickness and of $120 \mathrm{~km}$ radius and the observation point is supposed to be $100 \mathrm{~m}$ above the center of the circular water layer. Figure 6 also shows that, if we adopt the plain surface model in the near region, the errors seem to be within $0.4 \mu$ gal.

The elastic part $\left(g^{\mathrm{E}}\right)$ at a point $\mathrm{O}$ generated by a water prism as shown in Fig. 5 can be written as follows;

$$
\begin{aligned}
& g^{\mathrm{E}}=\frac{\rho g \beta(\lambda+2 \mu)}{4 \pi \mu(\lambda+\mu)} \int_{\eta_{1}}^{\eta_{2}} \int_{\xi_{1}}^{\xi_{2}} \frac{1}{\sqrt{\xi^{2}}+\eta^{2}} \mathrm{~d} \xi \mathrm{d} \eta \\
& =\frac{\rho g \beta(\lambda+2 \mu)}{4 \pi \mu(\lambda+\mu)}\left\{\xi \log \left(\sqrt{\xi^{2}}+\eta^{2}+\eta\right)+\eta \log \left(\sqrt{\xi^{3}+\eta^{2}}+\xi\right)-\xi\right\}||
\end{aligned}
$$




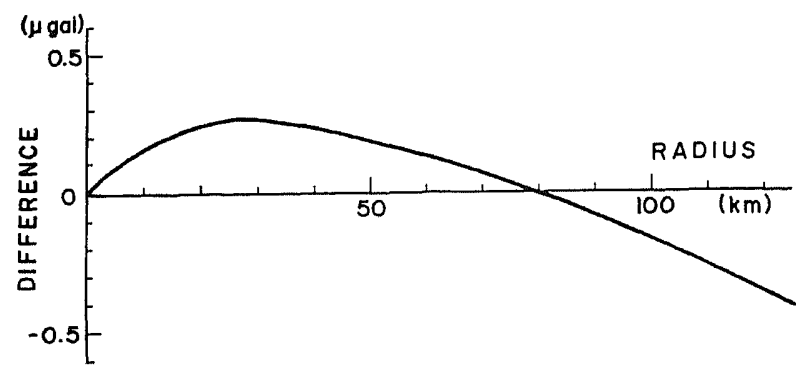

Fig. 7. Differences between the oceanic effects (elastic part) calculated by making use of a plain surface model and that of a spherical surface model. We assume here the effect is generated by a circular water layer of $1 \mathrm{~m}$ thickness and of $120 \mathrm{~km}$ radius and the observation point is supposed to be $100 \mathrm{~m}$ above the center of the circular water layer.

where $g$ is gravity at the observation point, $\lambda$ and $\mu$ Lame's moduli and $\beta$ the standard vertical gradient of gravity $(-0.3086 \mathrm{mgal} / \mathrm{m})$. This is well known as Boussinesq's solution (BoussinesQ, 1885). The notation \| in (2) is defined as follows:

$$
f(\xi, \eta) \|=f\left(\xi_{2}, \eta_{2}\right)-f\left(\xi_{1}, \eta_{2}\right)-f\left(\xi_{2}, \eta_{1}\right)+f\left(\xi_{1}, \eta_{1}\right) .
$$

In the case of a plain surface model, the elastic part generated by a water layer mass can also be obtained by summing up (2) over all the meshes in the near region. Figure 7 indicates the differences of the elastic part of oceanic effects between the plain surface model and the spherical surface model assuming a water layer of $1 \mathrm{~m}$ thickness within a radius of $120 \mathrm{~km}$. Here we use the Green's function given by FARrelL (1972) to calculate the elastic part in the case of the spherical surface model, while in the case of the plain surface model, we assume both $\lambda$ and $\mu$ to be $8.33 \times 10^{11} \mathrm{dyn} / \mathrm{cm}^{2}$, which minimize the errors due to adopting the plain surface model. Figure 7 also shows that, if we adopt the plain surface model for the near region, the errors due to such approximation seem to be within $0.3 \mu \mathrm{gal}$. Judging from the facts described above, it is sufficient to adopt the plain surface model for the near region.

In order to estimate sea level changes in any arbitrary location, the tidal charts are useful, although they seem to be insufficient for more precise estimation of sea level changes in the near region. Therefore, hourly tidal records obtained at 9 tidal stations in the near region (Fig. 2) are used in this paper. Fortunately, the tidal stations are distributed relatively uniformly around Izu peninsula, so that sea level at any point in the near region can be obtained from Briggs' two-dimensional interpolation method (BRIGGs, 1974) by making use of hourly tidal records. 
On the other hand, the sea surface in the far region is taken to be spherical. Let us consider a point A (observation station) and a fragment B (sea surface) on a spherical surface as shown in Fig. 8. Then the attraction part of oceanic effects at $A$ due to an uplift mass of sea water in $\mathrm{B}$ is approximately expressed as follows:

$$
g^{N}=-\frac{G \rho S h}{L^{3}} \cos \alpha,
$$

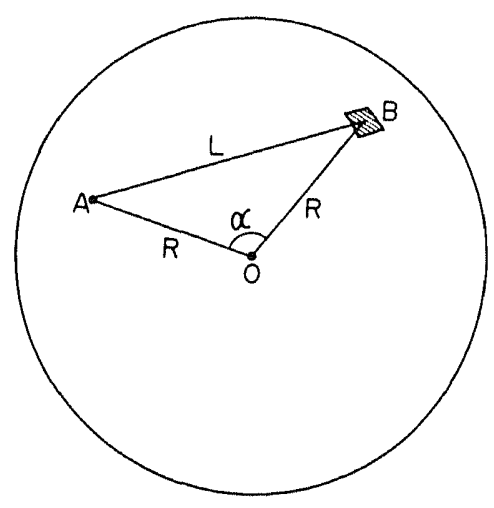

Fig. 8. An observation point $A$ and a fragment $B$ on a spherical earth. $R$, radius of the earth; $\mathrm{O}$, center of the earth.
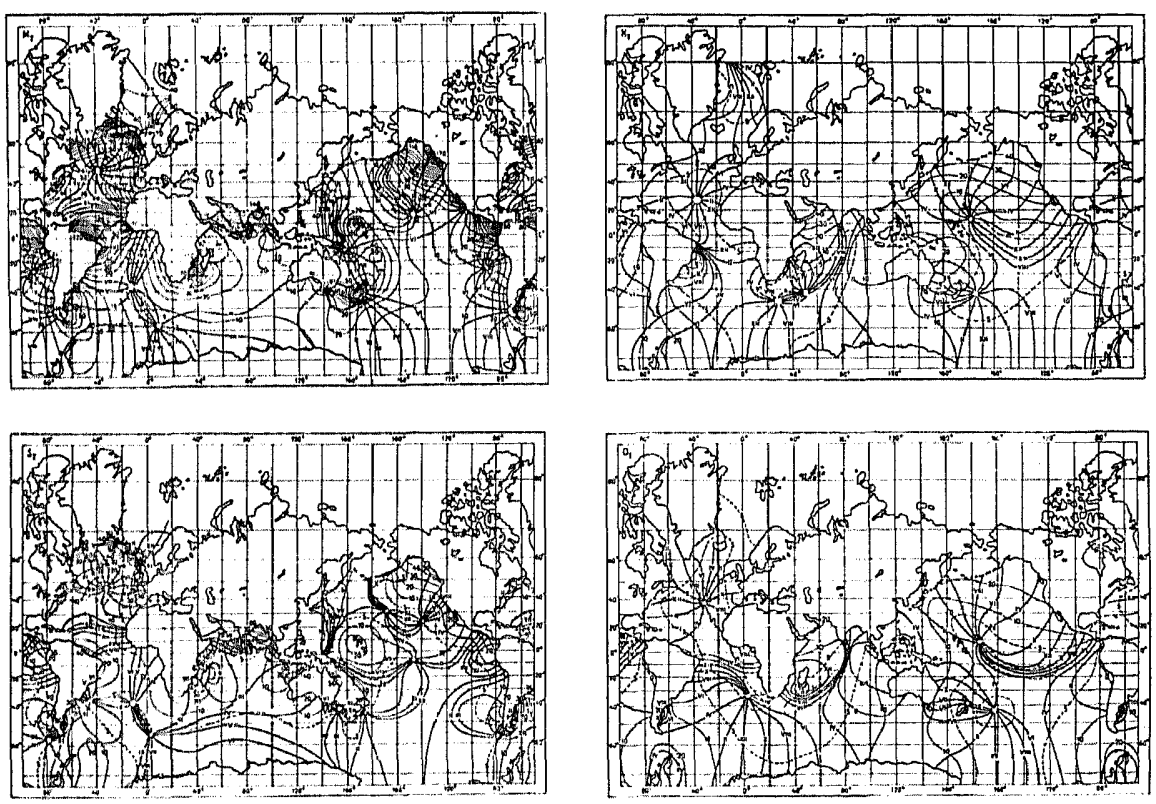

Fig. 9. Co-tidal charts of four principal constituents $\left(\mathrm{M}_{2}, \mathrm{~S}_{2}, \mathrm{~K}_{1}\right.$ and $\left.\mathrm{O}_{1}\right)$. Arabic numerals indicate the amplitude $(\mathrm{cm})$ and Roman numerals indicate the phase lag (hour) referred to Greenwich meridian (after TrRoN et al. (1967)). 

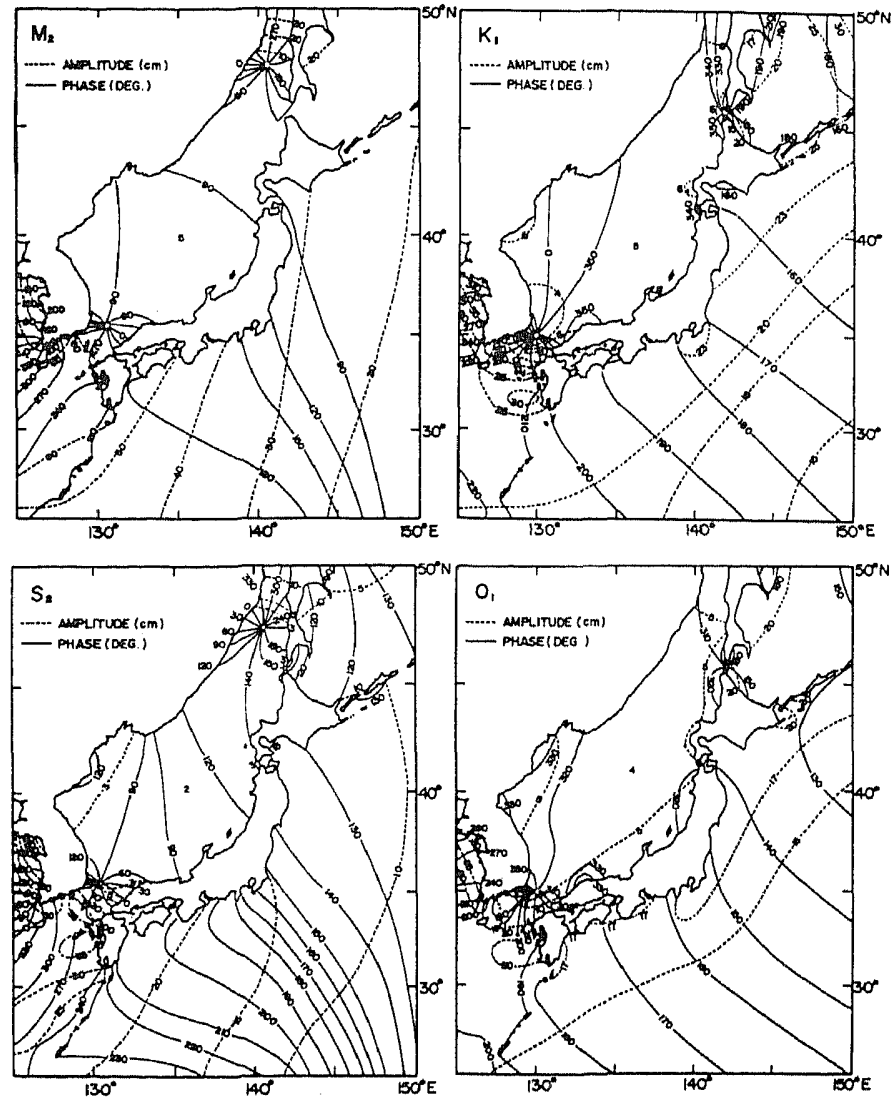

Fig. 10. Co-tidal charts of four principal constituents $\left(M_{2}, S_{2}, K_{1}\right.$ and $\left.O_{1}\right)$.

Phase lag is referred to $135^{\circ} \mathrm{E}$ (after Tsukamoto and NakAGawa (1979)).

where $S$ is the area of $\mathbf{B}, h$ the uplift of sea surface at the center of $\mathbf{B}$, $L$ the distance between $\mathrm{A}$ and the center of $\mathrm{B}$, and $\alpha$ the angular distance between $A$ and the center of $B$. The elastic part at $A$ due to an uplift mass of sea water in $\mathbf{B}$ is also approximately expressed as follows:

$$
g^{\mathrm{E}}=\rho \int_{S} g(\theta) h \mathrm{~d} s,
$$

where $g(\theta)$ is the Green's function obtained by Farrell (1972), $\theta$ the angular distance between $\mathrm{A}$ and $\mathrm{d} s$, and $h$ the uplift of sea surface in $\mathrm{d} s$.

Tidal charts used for ' $F$ ' and ' $G$ ' regions are those given by TIRON et al. (1967) and tidal charts used for ' $E$ ' region are those compiled originally by OGURA (1933) and modified by Tsukamoto and NAKAGAWA (1979). Figures 9 and 10 show the tidal charts used in this paper for four principal constituents, i.e. $\mathrm{M}_{2}, \mathrm{~S}_{2}, \mathrm{~K}_{1}$ and $\mathrm{O}_{1}$. 


\section{Results of Oceanic Tidal Corrections}

Figure 11 shows the location of gravity stations in Izu penisula. In precise gravity surveys for detecting crustal deformations in Izu peninsula, B.M. 9402 (Numazu) is taken as a base point (HAGIWARA et al., 1976a, 1976b, 1977). First, we estimate the oceanic effects on gravity at B.M. 9402 by making use of the method described in the previous section and the results are shown in the lower part of Fig. 12 as an example. In this figure, the broken line indicates the effect from the near region, the broken and dotted line the effect from the far region and the solid line the sum of them. The oceanic effect from the near region and that from the far region are almost equal and the total effects (sum of these two effects) amounts to about $10 \mu$ gal as shown in Fig. 12. Theoretical gravity tides of the solid earth at the site is also shown in the upper part of Fig. 12 as a reference. This calculation is based on the tables given by CARTWRIGHT and Tayler (1971) and Cartwright and Edden (1973). In the upper part of Fig. 12, the broken line indicates the sum of four constituents and

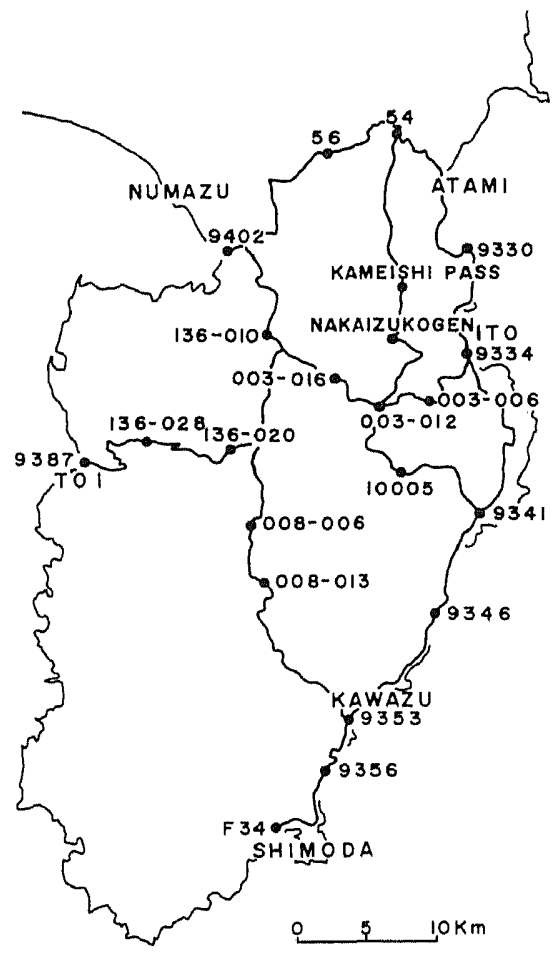

Fig. 11. Location of gravity stations in Izu peninsula (after Hagiwara et al. (1976b)). 


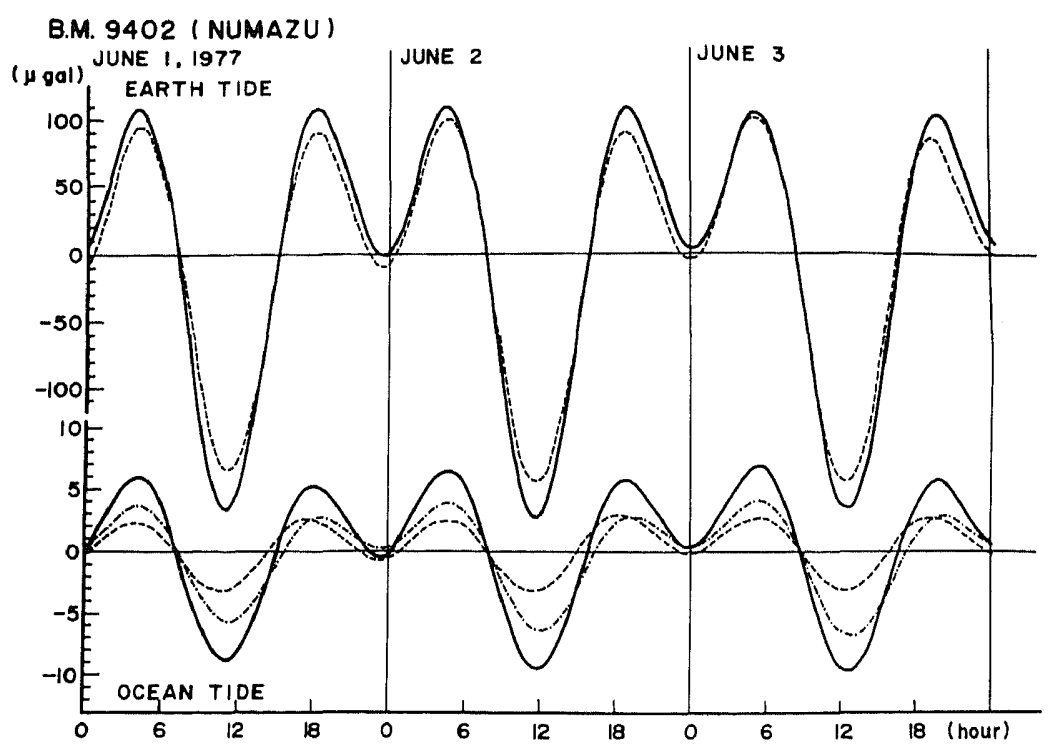

Fig. 12. Theoretical earth tides (upper) and oceanic tidal effects on gravity (lower) at B.M. $9402\left(35^{\circ} 2^{\prime} 30^{\prime \prime} \mathrm{N}, 138^{\circ} 54^{\prime} 21^{\prime \prime} \mathrm{E}\right)$, Numazu, Tokai District. Upper, broken line indicates the sum of four principal constituents and solid line the total earth tides; Lower, broken line indicates the effect from the near region, broken and dotted line the effect from the far region and solid line the sum of them.

the solid line the total earth tides (sum of all the constituents). Judging from this part of the figure, the difference between the sum of four principal constituents and the total earth tides is about 10 per cent of the total earth tides. Therefore, errors of oceanic effects on gravity tides due to applying the tidal charts of four principal constituents may be regarded as large as 10 per cent. In addition, considering the uncertainty of tidal charts and of the earth model, errors of oceanic effects might exceed 10 per cent of the total oceanic effects.

Next, we shall consider the spatial variation in oceanic effects. Figures 13 and 14 show the oceanic effects on gravity from the near and far regions respectively at each of the observation stations in Izu peninsula on the assumption that the whole sea surface rises uniformly by $1 \mathrm{~m}$. In both the figures, solid circles indicate the attraction part and open circles elastic part. Judging from these figures, the far-region effects are almost uniform at every gravity station in Izu peninsula. The differences among them are within $0.3 \mu \mathrm{gal}$. This fact indicates that the value of the farregion effect at one gravity station can be used uniformly for those at any other stations in Izu peninsula. On the contrary, the near-region effects 


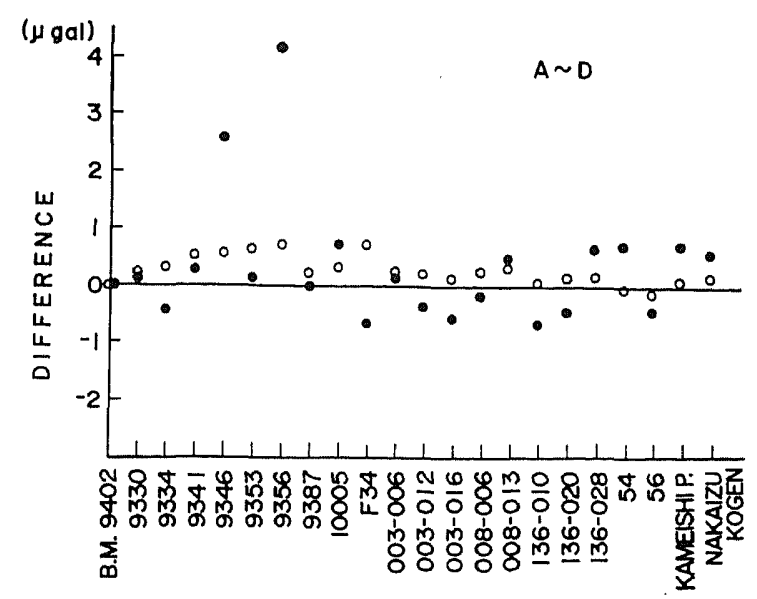

Fig. 13. Spatial variation in oceanic effect from the near region on gravity. The numbers in the abscissa are bench mark numbers in Izu peninsula.

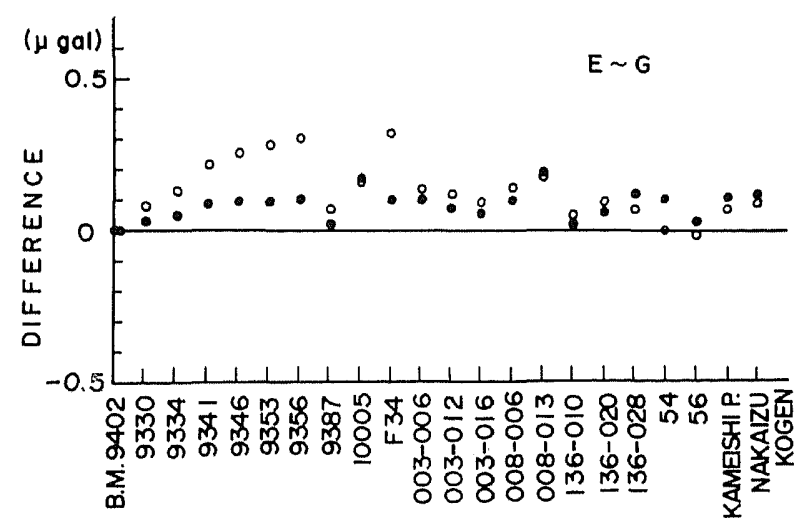

Fig. 14. Spatial variation in oceanic effect from the far region on gravity. The numbers in the abscissa are bench mark numbers in Izu peninsula.

are not so uniform, the difference sometimes amounting to about $5 \mu$ gal, so that the near-region effects at each of the gravity stations should be calculated individually. If we observe tidal gravity changes continuously at the base station during high-precision gravity resurveys for detecting the local crustal movement, we can correct the total effect of earth tides and oceanic tides for surveyed data, on the assumption that the earth tidal effect and the far-region oceanic tidal effect are almost uniform at any station, and these corrections are free from errors due to the uncertainty of tidal charts used for the global oceans. 


\section{G-Factor Estimation}

Earth tidal corrections are essentially important for gravity surveys, because their maximum values sometimes exceed $100 \mu \mathrm{gal}$. In order to estimate the solid earth tides, we must estimate the G-factor and phase lag precisely by continuous gravimetric observations. NAKagawA (1962) estimated the G-factor and phase lag to be about 1.20 (G-factor) and 0 hour (phase lag), respectively, on the basis of gravimetry at various stations in Japan.

In this paper, the G-factor and phase lag are estimated by another method which is based on the assumption that the optimal tidal corrections are made so as to minimize the standard deviation of surveyed data. In this case, the standard deviation of surveyed data on each day is expressed as follows (NAKAGAWA et al., 1973);

$$
\text { S.D. }=\sqrt{\frac{\sum_{i=1}^{n}\left\{g_{i}-\left(a t_{i}+b\right)\right\}^{2}}{n-2}}
$$

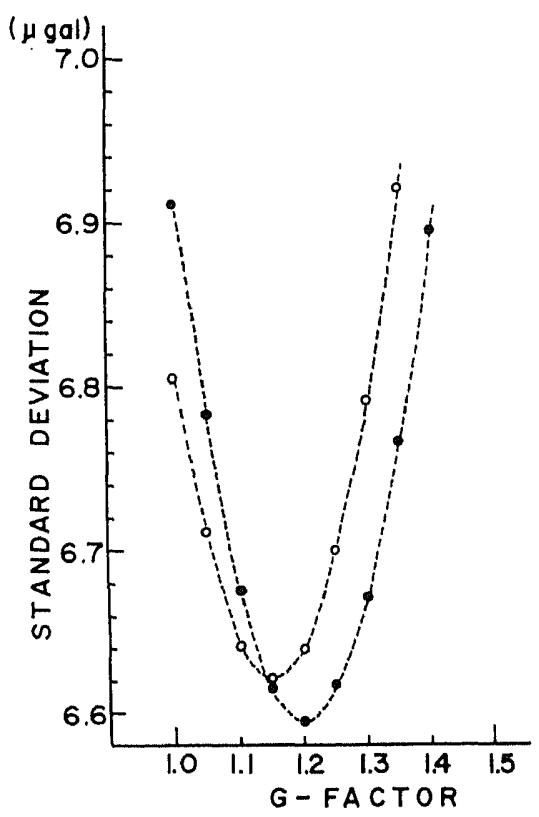

Fig. 15. Relation between the G-factor used in the earth tidal corrections and standard deviation of the measured gravity data. Open circles indicate the results after both oceanic and earth tidal corrections, and solid circles indicate the results after only earth tidal corrections. 


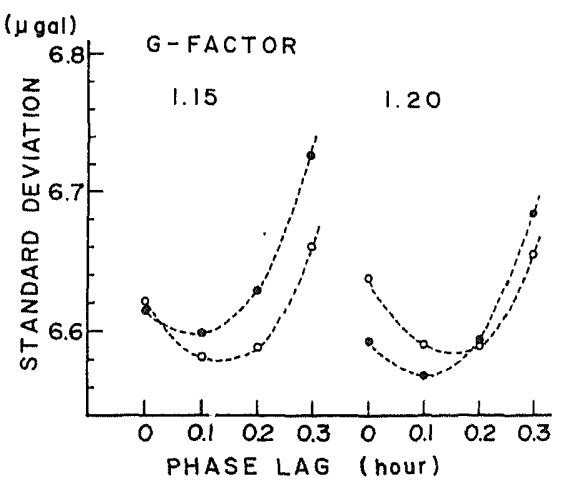

Fig. 16. Relation between the phase lag used in the earth tidal corrections and standard deviation of the measured gravity data. Open circles indicate the results after both oceanic and earth tidal corrections, and solid circles indicate the results after only earth tidal corrections.

where $g_{i}$ is the gravity value of the $i$-th measurement, $a$ and $b$ the drift coefficients, and $n$ the number of data. The G-factor and the phase lag used here are related to the total earth tides, not to each of their constituents. Data used in this paper have been obtained by six gravity surveys during the period from December, 1974 to June, 1977 in Izu peninsula (Hagiwara et al., 1976a, 1976b, 1977). The number of the data is 668 , which is sufficient for the statistical treatment.

First of all, we estimate the optimal G-factor used for the earth tidal corrections. Figure 15 shows the relation between the G-factor used in the earth tidal corrections and the standard deviations of the surveyed data. In this figure, open circles indicate the standard deviations of surveyed data after both earth and oceanic tidal corrections have been made, and solid circles indicate those after only earth tidal corrections have been made. The $\mathrm{G}$-factor which leads to the minimum standard deviation is about 1.15 when phase lag is supposed to be zero, and if oceanic tidal corrections are not made, its value is 1.20. The difference between the two factors results from the effects of oceanic tides.

Secondly, we estimate the optimal phase lag used for the earth tidal corrections. Figure 16 shows the relation between the phase lag used in the earth tidal corrections and the standard deviations of surveyed data in the two cases where the G-factor is 1.15 and 1.20. The phase lag which leads to the minimum standard deviation of surveyed data is about 0.1 hour in both cases. Judging form Figs. 15 and 16, the optimal Gfactor and phase lag used for the earth tidal corrections are 1.15 and 0.1 hour, respectively, and if oceanic tidal corrections are not made, their values become 1.20 and 0.1 hour, respectively. 


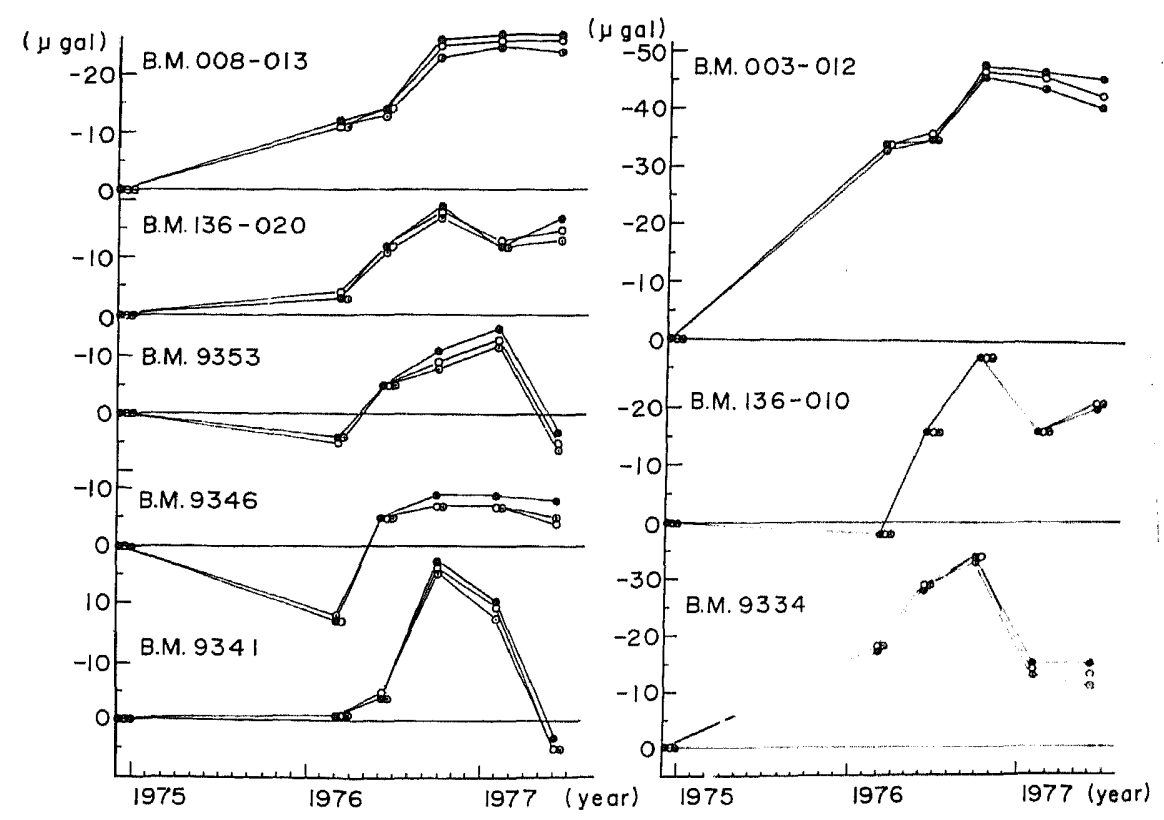

Fig. 17. Secular changes in gravity at 8 stations in Izu peninsula. Solid circles indicate the results after earth tidal corrections with G-factor of 1.2 and phase lag of 0 hour, double circles with 1.2 (G-factor) and 0.1 hour (phase lag) and open circles with 1.15 (G-factor) and 0.1 hour (phase lag).

After earth and oceanic tidal corrections have been made, the resultant gravity values are determined. In Fig. 17, temporal changes in gravity at 8 stations in Izu peninsula are shown (HAGIWARA et al., 1977). Three sets of tidal constants are used for the earth tidal corrections here, namely, the G-factor and phase lag being, respectively, 1.20 and zero (solid circles), 1.20 and 0.1 hour (double circles), and 1.15 and 0.1 hour (open circles). In the three cases described above, the temporal gravity changes are obtained within a scattering range of $5 \mu \mathrm{gal}$. Judging from these results, the errors due to the uncertainty of tidal constants of gravity seem to be less than about $5 \mu$ gal. These errors can also be overcome by continuous observations of gravity tides at the base station during gravity surveys for detecting the crustal movement.

\section{Concluding Remarks}

The effects of oceanic tides and earth tides on gravity are estimated for correcting gravity values in high-precision gravity resurveys. The detection of an accurate gravity change depends on how exactly these effects are corrected. It is essential to oceanic tidal corrections what 
version of tidal charts and earth model we adopt, and in case of earth tidal corrections, it is essential how exact are the values of the tidal gravity constants we adopt. The maximum errors due to such uncertainties amount to $5 \mu \mathrm{gal}$ or so. In conclusion, we recommend to make continuous observations of gravity tides at the base station together with hourly tidal records at the nearby tidal stations during gravity-change surveys.

The author wishes to express his gratitude to Associate Professor Y. Hagiwara for his helpful suggestions and critical review of the manuscript. He also wishes to thank Dr. H. Tajima, Mr. S. Izutuya, Dr. K. Nagasawa, Dr. I. Murata, Mr. M. Yanagisawa and Mr. S. Shimada for their helpful discussions. He also wishes to thank the personnel of Geographical Survey Institute, Japan Meteorological Agency and Hydrographic Division who were kind enough to send him the hourly tidal records, and wishes to thank Mr. H. Tsukamoto who was kind enough to send him the tidal charts.

\section{REFERENCES}

Boussinesq, J., Application des Potentiels a l'Etude de l'Equilibre et du Mouvement des Solides Elastiques, 508 pp, Gauthier-Villars, Paris, 1885.

BRIGGS, I. C., Machine contouring using minimum curvature, Geophysics, 39, 39-48, 1974.

Cartwright, D. E. and A. C. Edden, Corrected tables of tidal harmonics, Geophys. J. $R$. Astron. Soc., 33, 253-264, 1973.

CARTwRIGHT, D. E. and R. J. TAYLER, New computations of the tide-generating potential, Geophys. J. R. Astron. Soc., 23, 45-74, 1971.

FARRell, W. E., Deformation of the earth by surface loads, Rev. Geophys. Spase Phys., 10, 761-797, 1972.

Hagrwara, Y., Tidal groundwater-related gravity change in a seaside area, J. Geod. Soc. Japan, 23, 42-43, 1977.

Hagiwara, Y., H. Tajima, S. Izutuya, and M. Satomura, Gravity changes on Izu peninsula, J. Geod. Soc. Japan, 22, 17-22, 1976a (in Japanese).

Hagrwara, Y., H. Tajima, S. Izutuya, and H. Hanada, Gravity changes in the eastern part of Izu peninsula during the period of 1975-1976, J. Geod, Soc. Japan, 22, 201209, 1976b (in Japanese).

HaGiWARA, Y., H. TAJIMA, S. IzUTUYA, and H. HANADA, Gravity change associated with earthquake swarm activities in the eastern part of Izu peninsula, Bull. Earthq. Res. Inst., 52, 141-150, 1977 (in Japanese).

LAMBERT, A. and C. BEAUMONT, Nano variations in gravity due to seasonal groundwater movements: implications for the gravitational detection of tectonic movements, $J$. Geophys. Res., 82, 297-305, 1977.

Nakagawa, I., Some problems on time change of gravity, Parts 1 and 2, Bull. Disas. Prev. Res. Inst., Kyoto Univ., 53, 1-105, 1962.

Nakagawa, I., M. Satomura, Y. Fukuda, S. Nakai, T. Seto, K. Tajima, N. Inouchi, U. Hagiwara, H. Tajima, S. Izutuya, M. Yanagisawa, H. Hanada, Y. Tomoda, H. Fujnmoto, T. Furuta, and S. OOKAWA, On characteristics of LaCoste \& Romberg Gravimeters (Model G), J. Geod. Soc. Japan, 19, 100-112, 1973 (in Japanese).

NAKAI, S., On the characteristics of LaCoste \& Romberg Gravimeter G305, Proc. Int. Lat. Obs. Mizusawa, 15, 76-83, 1975 (in Japanese). 
OGura, S., The tides in the seas adjacent to Japan, Bull. Hydrogr. Depart. Imp. Jap. Navy, 7, 1933.

Oshima, H. and I. Yokoxama, Effects of oceanic tides on gravity survey, Abstracts of the 47th Meeting of the Geodetic Society of Japan, 13-14, 1977 (in Japanese).*

SATO, T., Effects of oceanic tides on earth tides (1) About gravity tides, Abstracts of the 44th Meeting of the Geodetic Society of Japan, 64-65, 1975 (in Japanese).*

TANakA, T., Effect of oceanic tides on gravity tides in Japan: the case of $\mathrm{M}_{\mathrm{a}}$ tide, Geophys. J. R. Astron. Soc., 50, 141-147, 1977.

Tanaka, T., A comment upon the effect of ocean tides on earth tides, Ann. Disas. Prev. Res. Inst., Kyoto Univ., 21, 137-143, 1978 (in Japanese).

Tiron, K. D., Y. N. Sergeev, and A. N. Michurin, Tidal charts for the Pacific, Atlantic and Indian Oceans, Vestn. Leningr. Univ., 24, 123-135, 1967.

Tsubokawa, I., The use of gravimetric survey for earthquake prediction, Rep. Earthq. Predict. Obs. center, 1, 53-57, 1970 (in Japanese).

Tsukamoto, H. and I. Nakagawa, On effects of oceanic tides upon gravimetric tides for diurnal constituents, J. Geod. Soc. Japan, 24, 226-233, 1979.

* The title of the paper and the name of the proceedings were translated in English by the author. 